\title{
Estudio del glicoconjugado glucosa/manosa en mucosa gástrica de cerdos diagnosticados con Helicobacter pylori
}

\author{
Vandeerveen, M.P.'; Giménez, S.R.'; Grosso, M.C.'; Bonino, F.U. ${ }^{2}$; Macloughlin V.H. ${ }^{1}$ \\ ${ }^{1}$ Cátedra Histología, Fac. Agron.Vet., Univ.Nac.Río Cuarto, Córdoba, Argentina. \\ ${ }^{2}$ Cátedra Bioestadíst. FAV, UNRC. E-mail: mariapaulavdv@ayv.unrc.edu.ar
}

\begin{abstract}
Resumen
Vandeerveen M.P.; Giménez, S.R.; Grosso, M.C.; Bonino, F.U.; Macloughlin, V.: Estudio del glicoconjugado glucosa/manosa en mucosa gástrica de cerdos diagnosticados con Helicobacter pylori. Rev. Vet. 32: 1, 37-42, 2021. Helicobacter sp, bacilo Gram negativo, se encuentra asociado a gastritis, úlcera y cáncer gástrico.La prevalencia mundial de la infección por $H$. pylori es mayor al $50 \%$, constituyendo un problema de salud pública. Diferentes especies de Helicobacter afectan tanto a animales como seres humanos, pudiendo ser responsables de zoonosis. El proceso de colonización de esta bacteria depende en gran medida de su unión a glicoconjugados presentes en la mucosa gástrica del hospedero. El cerdo representa el modelo animal de elección para el estudio de la infección por esta bacteria. El objetivo del presente trabajo fue analizar el comportamiento del glicoconjugado glucosa/manosa en la mucosa gástrica de cerdos afectados por gastritis producida por Helicobacter $\mathrm{sp}$. Se utilizaron muestras de la región antral del estómago de cerdos mestizos, obtenidas de frigoríficos de Río Cuarto (Argentina). Dichas muestras fueron clasificadas en distintos grupos de estudio de acuerdo al tipo de gastritis y a la presencia y/o ausencia de Helicobacter $s p$. Las muestras fueron luego sometidas a lectin-histoquímica para la marcación del glicoconjugado glucosa/manosa y posteriormente evaluadas mediante microscopía óptica y análisis estadístico. Los resultados obtenidos indican que no hay diferencias entre los grupos en cuanto a la expresión de glucosa/manosa en epitelio y glándulas gástricas, pero sí se presentan diferencias significativas en la expresión de este glicoconjugado en lamina propia entre el grupo gastritis aguda positivo a Helicobacter $s p$ con respecto a los grupos gastritis aguda negativo a Helicobacter $s p$. y grupo con mucosa normal. Los resultados estadísticos revelan que existe relación entre el aumento en la marcación de glucosa/manosa y la presencia de Helicobacter $s p$ en lamina propia de mucosa gástrica de cerdos.
\end{abstract}

Palabras clave: Helicobacter pylori, cerdos, mucosa gástrica, lectin-histoquímica, glucosa / manosa.

\begin{abstract}
Vandeerveen, M.P.; Giménez, S.R.; Grosso, M.C.; Bonino F.U.; Macloughlin, V.: Study of the glucose/mannose glycoconjugate in the gastric mucosa of pigs diagnosed with $\mathrm{He}$ licobacter pylori. Rev. Vet. 32: 1, 37-42, 2021. Helicobacter pylori, a Gram negative bacillus, is associated with gastritis, ulcer, and gastric cancer. The worldwide prevalence of $H$. pylori infection is greater than $50 \%$, constituting a public health problem. Different species of $\mathrm{He}$ licobacter were described, which affect both animals and humans, and may be responsible for zoonoses. The colonization process of this bacterium depends largely on its binding to glycoconjugates present in the gastric mucosa of the host. The pig represents the animal model of choice for the study of infection by this bacterium. The objective of the present work was to analyze the behavior of the glucose/mannose glycoconjugate in the gastric mucosa of pigs affected by gastritis caused by Helicobacter $s p$. Samples from the antral region of the stomach of crossbred pigs were used, obtained from slaughterhouse in the Río Cuarto (Argentina). The samples were classified into different study groups according to the type of gastritis and the presence or absence of Helicobacter sp. A lectin histochemistry study for glucose/mannose glycoconjugate was carried out, the evaluated under microscope and statistical analysis performed. The results obtained indicate that there were no significant differences between the groups in the glucose/mannose expression in epithelium and gastric glands, but there were significant differences in the expression of this glycoconjugate in lamina propria between Helicobacter $s p$ positive acute gastritis with respect to the Helicobacter $s p$ negative acute gastritis groups and with normal mucosa group. This results reveal that
\end{abstract}


there is relationship between the increase in glucose/mannose expression and the presence of Helicobacter $s p$ in the lamina propria of pig gastric mucosa.

Key words: Helicobacter pylori, pigs, gastric mucosa, lectin-histochemistry, glucose / mannose.

\section{INTRODUCCION}

Helicobacter pylori, bacilo Gram negativo, fue declarado como la principal causa de úlcera péptica y cáncer en seres humanos por la Organización Mundial de la Salud en el año 1994. La prevalencia mundial de la infección por $H$. pylori es mayor al $50 \%$, constituyendo un problema de salud pública ${ }^{20}$.

Se han descrito especies del genero Helicobacter en una amplia variedad de huéspedes animales. Cuando estas bacterias son identificadas por su morfología y no a nivel de especie, se utiliza el término Helicobacter no H. pylori "non-H. pylori helicobacters" (NHPH) ${ }^{9}$. El porcentaje de pacientes infectados con $\mathrm{NHPH}$ varía entre $0.2 \%$ y $6 \%{ }^{5}$. La infección con estos NHPH en forma de espiral, en seres humanos se ha asociado con gastritis, ulceración gástrica y linfoma MALT gástri$\mathrm{CO}^{12}$

El aislamiento de Helicobacter sp de varios animales, que en su mayoría viven en entornos humanos, los hace sospechosos de albergar $H$. pylori en sus estómagos y por lo tanto participar en la transmisión de este patógeno ${ }^{14}$. Vivir en proximidad cercana con gatos, perros y en especial cerdos, se ha identificado como un factor de riesgo para estas infecciones ${ }^{10}$. Además, algunos autores consideran a Helicobacter como bacteria patógena emergente transmisible por los alimentos ${ }^{11}$.

En cerdos la prevalencia de Helicobacter suis en el estómago al momento del sacrificio varía del 8 al $95 \%{ }^{4,5}$. Investigaciones mostraron que de las infecciones humanas con NHPH del 13,9\% al 30,9\% corresponden a $H$. suis ${ }^{30}$, siendo después del $H$. pylori el más frecuente en seres humanos ${ }^{16}$.

En porcinos se ha logrado reproducir úlcera gástrica mediante la inoculación experimental con H. pylo$r{ }^{1}$. La similitud morfológica entre la úlcera porcina y la humana no implica una patogénesis similar, pero sugiere la presencia de mecanismos comunes para su desarrollo ${ }^{13}$.

Se han propuesto varios factores de virulencia para H. pylori, algunos de ellos asociados al proceso de colonización y otros sindicados con el daño en la mucosa gástrica ${ }^{29}$. En el proceso de colonización, la capa de moco es la primer barrera que los patógenos gástricos deben superar. Los componentes principales de la capa mucosa son glicoproteínas de mucina densamente glucosiladas ${ }^{24}$. H. pylori se fija a la superficie o a las uniones intercelulares del epitelio gástrico generando daños en el mismo ${ }^{15}$

La lamina propia adyacente atrae células defensivas, infiltrándose con polimorfonucleares y mononucleares ${ }^{15}$. La inflamación crónica disminuye la concen- tración de células D (productoras de somatostatinas) y la hipergastrinemia resultante, incrementa la secreción de jugo gástrico. El desbalance entre factores agresivos (incrementados) y defensivo (disminuidos), pueden expresarse como una lesión ulcerada ${ }^{6}$.

Los glicoconjugados se encuentran en diferentes componentes del tracto gastrointestinal, entre ellas las células de la mucosa gástrica ${ }^{\top}$. Poseen una amplia variedad de funciones como: intervención en el proceso de reconocimiento célula-célula, protección de las células epiteliales contra valores de $\mathrm{pH}$ extremos o de las enzimas hidrolíticas, unión e inhibición de células citotóxicas, entre otras ${ }^{8}$, cumpliendo así un papel importante en la protección del epitelio, aunque pudiendo también facilitar la adhesión de bacterias al mismo ${ }^{21}$.

El patrón de glicosilación puede sufrir modificaciones debido tanto a cambios fisiológicos como a procesos patológicos. El conocimiento del patrón de glicosilación en condiciones normales es esencial para evaluar sus modificaciones en diversas patologías y tener mayor comprensión de su patogenie. Las lectinas constituyen una herramienta fiable para ello ${ }^{21}$.

El uso de los modelos animales para el estudio de la helicobacteriosis ha sido amplio a nivel mundial, lo cual ha permitido grandes avances en cuanto al conocimiento de la epidemiologia, infección y patogenia de Helicobacter en la enfermedad gástrica humana ${ }^{19}$.

El cerdo -por su condición anatómica- presenta mucha similitud con el ser humano, siendo el modelo de elección para el estudio de la infección por Helicobacter, su interacción con otros agentes patógenos virusbacteria y la carcinogénesis gástrica ${ }^{22}$.

El objetivo del presente trabajo fue analizar el comportamiento del glicoconjugado glucosa/manosa a través de la técnica de lectinhistoquímica en la mucosa gástrica de cerdos afectados por gastritis producida por Helicobacter $s p$.

\section{MATERIAL Y MÉTODOS}

La toma de muestras de estómago de cerdos $(n=50)$, su procesamiento por la técnica histológica convencional y el posterior diagnóstico de gastritis (crónicas/agudas) y presencia/ausencia de Helicobacter $s p$ son parte de un trabajo previo realizado por este mismo grupo de investigación ${ }^{30}$.

En todos los casos se trató de animales destinados a faena y considerados libres de enfermedad de acuerdo al examen clínico y post-mortem realizado. El trabajo contó con el aval del Comité de Ética de la Universidad Nacional de Río Cuarto (Córdoba). 
Las muestras fueron clasificadas en diferentes grupos de estudios:

Grupo A: cerdos $\mathrm{G} / \mathrm{H}+$ : cerdos con gastritis agudas y presencia de Helicobacter $s p$.

Grupo B: cerdos G/H-: cerdos con gastritis agudas sin presencia de Helicobacter $s p$.

Grupo C: cerdos $\mathrm{G} / \mathrm{H}+$ : cerdos con gastritis crónicas y presencia de Helicobacter $s p$.

Grupo D: cerdos G/H-: cerdos con gastritis crónicas sin presencia de Helicobacter sp.

Grupo E: cerdos con mucosas gástricas normales

Para la determinación del glicoconjugado glucosa/ manosa en la mucosa gástrica a través de la técnica de lectinhistoquímica, se utilizó como marcador la lectina vegetal biotinilada con canavalina $\mathrm{A}$ (con A). Como reveladores: el complejo universal avidina-biotinaperoxidasa $(\mathrm{ABC})$ y como substrato de la enzima: la diaminobencidina (DAB).

Las muestras fueron observadas con un microscopio Orthoplan. Se realizó la adquisición de imágenes a través de una cámara digital. Carl Zeiss AxioCam ERc $5 s$.

Los resultados obtenidos en la técnica lectínhistoquímica fueron expresados en forma cualitativa determinando que: (-): negativo, $( \pm)$ : pobre marcación, $(+)$ : moderada marcación, $(++)$ : abundante marcación, $(+++)$ : cuantiosa marcación.

Posteriormente, para el análisis cuantitativo, se determinó la distribución de la intensidad de inmunomarcación a través del valor de High Score (HS), donde para cada muestra, el valor HS derivó de la sumatoria de los porcentajes de tejido epitelial gástrico marcado para cada intensidad, multiplicado por el ponderado para esa intensidad de marcación. High Score $=\sigma p i$ $(i+1) . i=$ intensidad de marcación. $p i=\%$ de tejido marcado para cada intensidad.

En cada preparado, cinco áreas seleccionadas al azar fueron evaluadas bajo un microscopio de luz con un aumento de $40 \mathrm{X}$, utilizando la puntuación media ${ }^{28}$.

\section{RESULTADOS}

En el Grupo A se apreció una pobre marcación epitelial (+/-), una lámina propia con una marcación homogénea que va de abundante $(++)$ a cuantiosa $(+++)$ y glándulas con una marcación moderada $(+)$ a cuantiosa $(+++)$ de distribución heterogénea (Figura 1-A).

Las muestras correspondientes a mucosa gástrica pertenecientes al Grupo B, revelaron la presencia del glicoconjugado glucosa/manosa en epitelio con una marcación pobre $(+/-)$ en la región apical a moderada $(+)$ y en algunos casos abundante $(++)$ en la región basal. En lamina propia la marcación fue moderada $(+)$ y de distribución uniforme.

Las glándulas presentaron una marcación de moderada (+) a abundante (++) (Figura 1-B). En el Grupo $\mathbf{C}$ se observó un epitelio con pobre marcación (+/-) en la región apical, a moderada $(+)$ en la región basal. La lamina propia presentó una marcación que variaba de abundante $(++)$ a cuantiosa $(+++)$, mientras que las glándulas presentaron una marcación moderada $(+)$ a abundante $(++)$, siendo la distribución uniforme en ambos casos (Figura 1-C).

Las muestras gástricas de cerdos del Grupo D presentaron en el epitelio zonas con una pobre marcación $(+/-)$ y zonas con marcación cuantiosa $(+++)$ distribuidas heterogéneamente. La lamina propia, al igual que las glándulas, presentaron una marcación moderada $(+)$ (Figura 1-D).

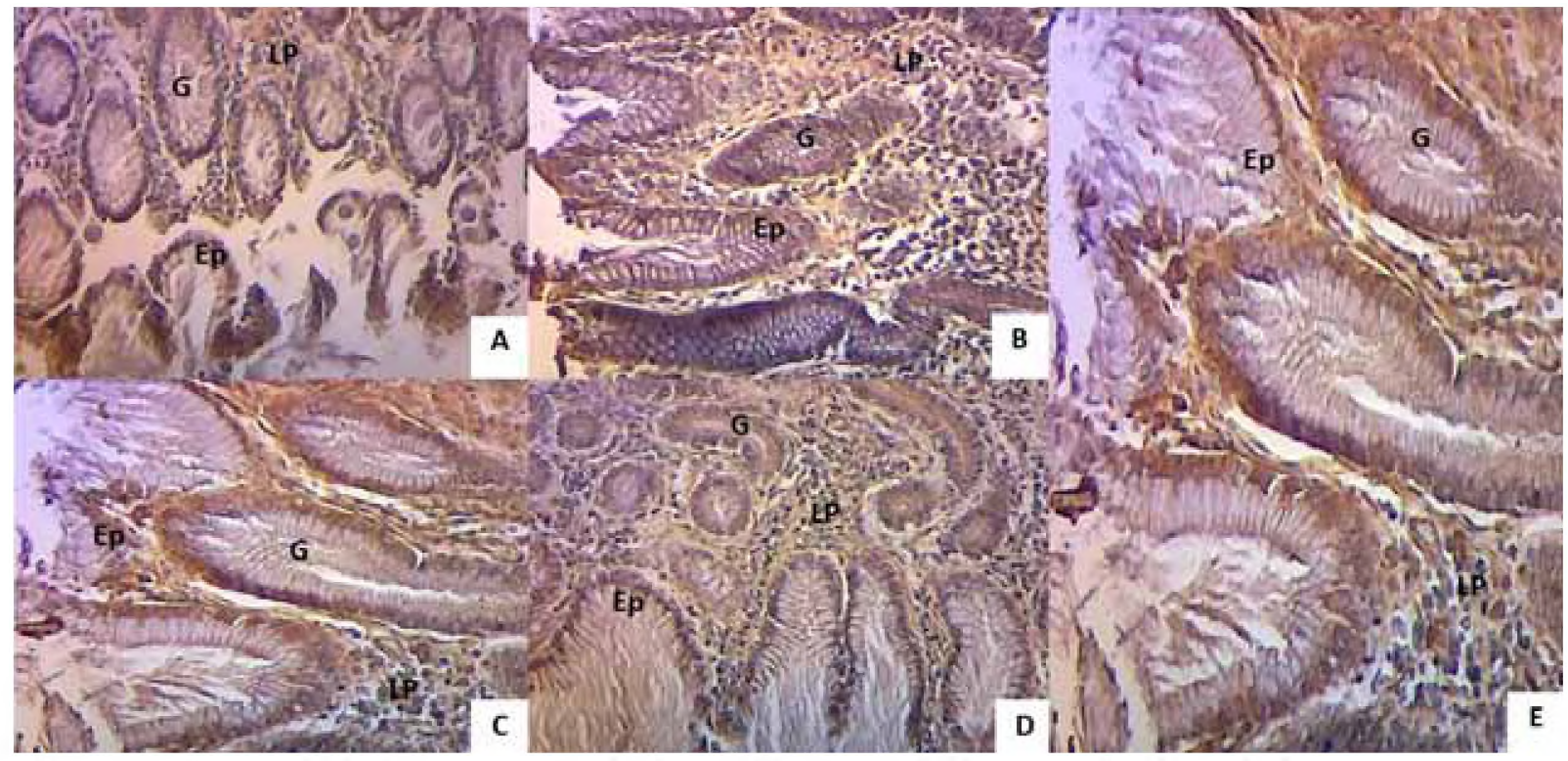

Figura 1. Microscopía óptica de muestra gástrica, región antral, técnica de lectin-histoquímica con A 400x. A: mucosa gástrica Grupo A. B: mucosa gástrica Grupo B. C: mucosa gástrica Grupo C. D: mucosa gástrica Grupo D. E: mucosa gástrica Grupo E. Ep: epitelio. LP: lamina propia. G: glándulas 


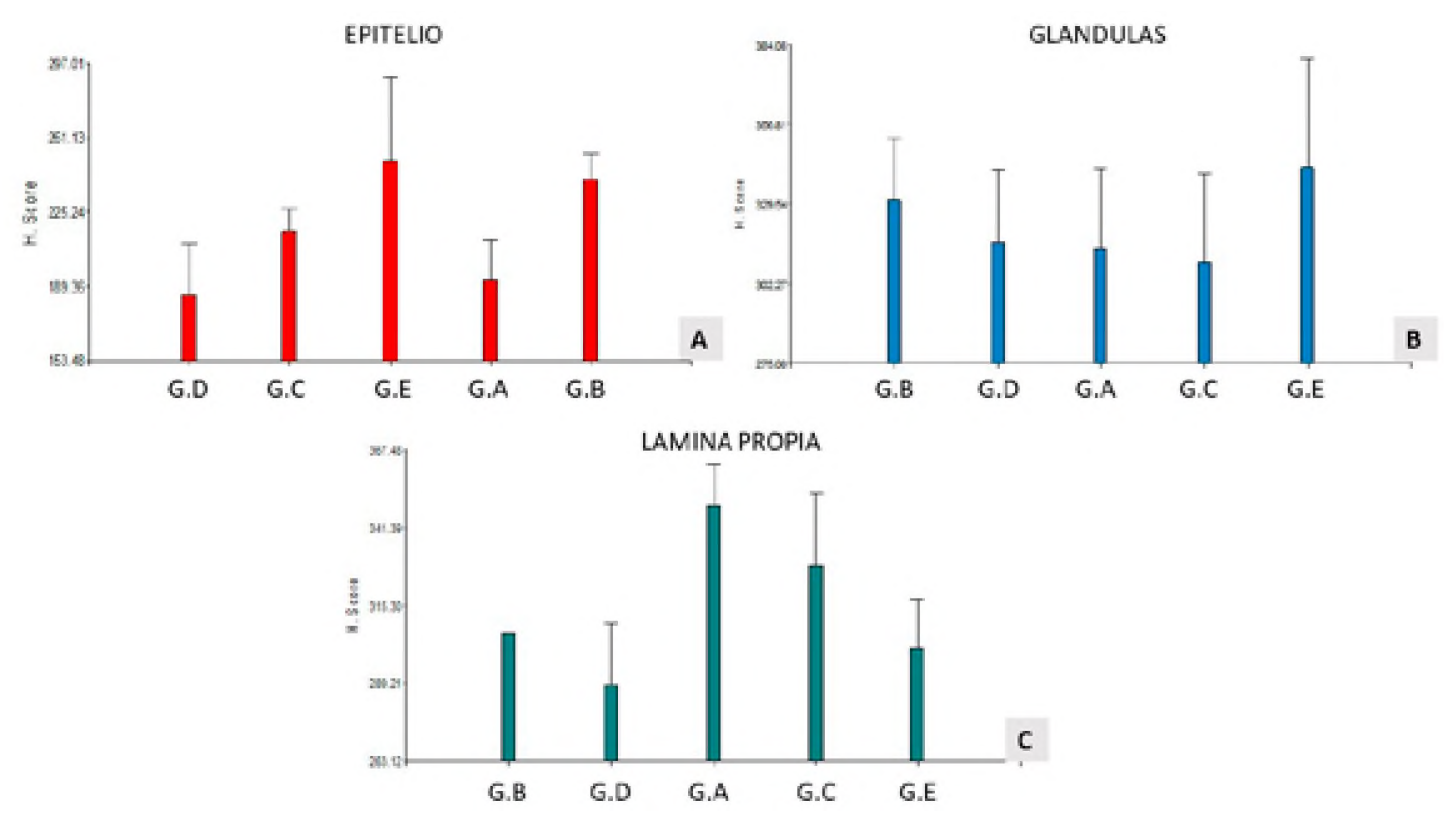

Figura 2. Valores medios de la variable HS para Lectina Con A con sus respectivas desviaciones estándar. A: epitelio. B: glándulas. C: lámina propia. G.A: grupo A. G.B: grupo B. G.C: grupo C. G.D: grupo D. G.E: grupo E.

En la mucosa gástrica antral normal (Grupo E), se pudo visualizar una marcación cuantiosa $(+++)$ y uniforme del epitelio y las glándulas, así como una tinción abundante (++) de la lámina propia (Figura 1-E).

Cuando se evaluó mediante el análisis estadístico la intensidad de marcación del epitelio en los diferentes grupos, no se encontraron diferencias estadísticamente significativas en la expresión del glicoconjugado glucosa/manosa (Figura 2-A). En cuanto a las glándulas, el análisis estadístico reveló una distribución homogénea de la marcación en los diferentes grupos cuyas diferencias no eran estadísticamente significativas (Figura 2-B).

En relación a la lámina propia, el estudio estadístico (Figura 2-C) evidenció diferencias significativas entre el Grupo A y el Grupo E, existiendo una marcación cuantiosa del primero en relación al segundo grupo. Diferencias significativas también se presentaron entre el Grupo A y el Grupo B, presentándose como en el caso anterior una marcación cuantiosa en el Grupo A en relación al Grupo B.

\section{DISCUSIÓN}

Los hidratos de carbono en las superficies celulares desempeñan un papel clave en el proceso de reconocimiento de célula-célula, y en la adhesión de los microorganismos a las células huésped a través de proteínas de unión a carbohidratos llamados lectinas ${ }^{2}$ como ocurre con Helicobacter $s p^{25}$.

Por lo tanto, conocer el patrón de glicosilación es de alta relevancia fisiopatológica durante el proceso de infección ${ }^{21}$. Debido a la diversidad de especies de $\mathrm{He}$ licobacter y a su capacidad para colonizar de manera eficiente el estómago de mamíferos, se ha propuesto que estas bacterias podrían causar lesiones gástricas en animales de forma análoga a las producidas en el ser humano ${ }^{26,27}$.

La similitud morfológica entre la úlcera porcina y la humana, sugiere mecanismos comunes para su desarrollo, esto sumado a las características anatómicas semejantes entre ambas especies, hacen del cerdo el modelo de elección para el estudio de la infección por Helicobacter ${ }^{22,26}$.

Existen pocos registros de trabajos realizados en animales que aborden el estudio del patrón de glicosilación en mucosa gástrica con Helicobacter spp, especialmente a través de la lectina Con $A$.

En especies como el cobayo, se analizó el patrón de expresión de los residuos de azúcar de la mucosa gástrica infectada por $H$. pylori, diagnosticada con gastritis crónica activa y no infectada mediante las lectinas Triticum vulgaris (WGA), Sambuccus nigra (SNAI), Ulex europaeus (UEA-I), Helix pomatia (HPA) y Phaseolus vulgaris (PHA-L).

Aunque se encontraron intensidades de tinción heterogéneas para las mismas lectinas, no se hallaron diferencias significativas entre los grupos. Sin embargo, los patrones de glucosa/manosa no fueron estudiados mediante la lectina Con $\mathrm{A}^{17}$.

En seres humanos se estudiaron a través de lectinhistoquímica, muestras de biopsias de mucosa gástrica normal y diagnosticadas con gastritis crónica activa e infectadas con $H_{\text {. pylori }}{ }^{3}$. Observaron diferencias significativas entre las reacciones de tinción de glicoconjugados para las lectinas UEA-I, Concanavalina A succinilada (SCA) y Arachis hypogaea (PNA).

La tinción para glucosa/manosa (unida por SCA) fue negativa en el moco extracelular y la membrana apical del epitelio de la mucosa normal, mientras que 
las mismas regiones fueron positivas en mucosa infectada por H. pylori.

Investigadores ${ }^{18}$ analizaron mediante microscopia óptica y microscópica de fluorescencia, la capa mucosa superficial de biopsias gástricas humanas diagnosticadas con gastritis crónica activa positivos a $H$. pylori y mucosas normales mediante las lectinas WGA y Con A.

Los resultados indicaron para la lectina Con A un patrón de unión intensa en el grupo mucosa normal, mientras que la unión fue débil a negativa en el grupo positivo a $H$. pylori. Por otro lado, el patrón de unión fue difuso para la lectina WGA en ambos grupos. Estos resultados sugieren un cambio significativo en los restos de hidratos de carbono presentes en la superficie de las células gástricas durante la infección ${ }^{18,23}$.

Los resultados obtenidos en nuestro estudio indican diferencias estadísticamente significativas en la expresión de glucosa/manosa solo en lámina propia, entre los grupos gastritis aguda Helicobacter $(+)$ y mucosa normal, observándose una marcación cuantiosa en el primero con respecto al segundo y entre los grupos gastritis aguda Helicobacter $(+)$ y gastritis aguda $\mathrm{He}$ licobacter $(-)$ presentándose como en el caso anterior una marcación mayor en el grupo gastritis aguda Helicobacter $(+)$.

En conclusión, los resultados estadísticos de este estudio revelan que existe relación entre el aumento en la marcación de glucosa/manosa y la presencia de $\mathrm{He}$ licobacter spp. en lamina propia de mucosa gástrica de cerdos.

\section{REFERENCIAS}

1. Akopyants NS, Eaton KA, Berg DE. 1995. Adaptative mutation and colonization during Helicobacter pylori infection of gnotobiotic piglets. Infect Imm 63: 116-121.

2. Artis D, Grencis RK. 2008. The intestinal epithelium: sensors to effectors in nematode infection. Mucosal Immun 1: 4, 252-264.

3. Baczako K, Kuhl P, Malfertheiner P. 1995. Lectin-binding properties of the antral and body surface mucosa in the human stomach are the difference revelant for Helicobacter pylori affinity?. Journal Pathol 176: 77-86.

4. Baele M et al. 2008. Isolation and characterization of Helicobacter suis sp. nov. from pig stomachs. Int J Syst Evol Microbiol 58: 1350-1358.

5. Baele $\mathbf{M}$ et al. 2009. Non-Helicobacter pylori helicobacters detected in the stomach of humans comprise several naturally occurring Helicobacter species in animals. FEMS Immun Med Microbiol 55: 306-313.

6. Debenedetti MA et al. 2018. Determinación citoinmunohistoquímica de células D en estómago de cerdos con Helicobacter spp. Ab Intus 1: 2, 39-46.

7. Delacruz $\mathbf{J}$ et al 2001. Application of lectins for the identification of glycoconjugate in the large intestine of chinchilla (Chinchilla lanigera). Biocell: 25: 1, 139

8. Gimeno EJ, Barbeito CG. 2004. Glicobiología, una nueva dimensión para el estudio de la biología y de la patología. Anales Acad Nac Agron \& Vet LVIII 58: 6-34.
9. Guendulain C, Sibilla ML. 2018. Helicobacter gástricos en perros y gatos y su significancia en la salud humana. $A b$ Intus 2: 1, 93-100.

10. Haesebrouck F et al. 2009. Gastric Helicobacters in domestic animals and nonhuman primates and their significance for human health. Clin Microbiol Rev 22: 2, 202-223.

11. Hernández PE. 2010. Bacterias patógenas emergentes transmisibles por los alimentos. http://www.analesranf. com/index.php/mono/article/viewFile /1111/1128

12. Joosten M et al. 2013. Case report: Helicobacter suis infection in a pig veterinarian. Helicobacter 18: 392-396.

13. Krakowka S, Eaton KA, Rings DM. 1995. Occurrence of gastric ulcer in gnotobiotic piglets colonized by Helicobacter pylori. Infec Immun 63: 2352-2355.

14. Kusters JG, Vliet AM, Kuipers EJ. 2006. Pathogenesis of Helicobacter pylori infection. Clin Microbiol Rev 19: 3, 449-490

15. León BR, Recavarren S, Ramirez RA. 1991. El aporte peruano a la investigación sobre Helicobacter pylori. Rev Méd Herediana 4: 173-181.

16. Liang $\mathbf{J}$ et al. 2013. Sequence typing of porcine and human gastric pathogen Helicobacter suis. Int $J$ Syst and Evol Microbiol 51: 3, 920-936.

17. Lueth $\mathbf{M}$ et al. 2005. Lectinhistochemistry of the gastric mucosa in normal and Helicobacter pylori infected guinea-pigs. J Mol Hist 36: 51-58

18. Melo MR, Cavalcanti C, Pontes FN, Carvalho L, Beltrao E. 2008. Patrones de tinción de lectina en la mucosa gástrica humana con y sin exposición a Helicobacter pylori. J Braz Microbiol 39: 2, 238-240.

19. Morales BA, Bermúdez V. 2008. Modelos animales para el estudio de la infección por el género Helicobacter en humanos. Rev Soc Med Quir Hosp Emerg Perez de León 39: 1, 30-33.

20. Morales MR, Castillo RG, López VY, Cravioto A. 2019. Helicobacter pylori. http://www.biblioweb.tic.unam.mx/ libros/microbios/Capll/. ISBN 968-36-8879-9.

21. Paredes LE. 2013. Glicoconjugados en epitelios. Sistema de Revisión en Investigación Veterinaria de San Marcos UPG, 1-9.

22. Polanco R et al. 2006. Lesiones gástricas asociadas a la presencia de bacterias del Género Helicobacter en caninos. Rev Cientif FCV-LUZ 6: 16, 585-592.

23. Quintana MP, Padra M, Padra JT, Benktander J, Linden SK. 2018. Mucus pathogen interactions in the gastrointestinal tract of farmed animals. Micro-organisms 6 : E55.

24. Rivas TF, Hernandez F. 2000 . Helicobacter pylori: factores de virulencia, patología y diagnóstico. Rev Biomed: 11: 187-207.

25. Rodríguez B, Aranzazu D, Ortiz L. 2008. Determinación de Helicobacter spp en cerdos en el depto.de Antioquia, Colombia. Rev Colom Cien Pec 21: 2, 210-218.

26. Rodríguez B, Aranzazu D, Ortiz L. 2009. Association of gastric ulcer and Helicobacter sp in pigs in Antioquia, Colombia. Rev Colom Cien Pec 22: 54-60.

27. Selam B, Kayisli UA, Mulayim N, Arici A. 2001. Regulation of fas ligand expression by estradiol and progesterone in human endometrium. Biol Reprod 65: 4, 979-985. 
28. Torres F, Torres C. 2016. Fisiopatología molecular en la infección por Helico-bacter pylori. Salud Uninorte 32: 3, $500-512$.

29. Vandeerveen MP et al. 2018. Determinación de L-fucosa en epitelio gástrico de cerdos con Helicobacter $s p$. Estudio preliminar. Morfovitual 2018. http:/www.morfo-virtual 2018. sld.cu/index.php/morfovirtual/2018/paper/view/212
30. Vandeerbulck K et al. 2005. Identification of non-Helicobacter pylori spiral organisms in gastric samples from humans, dogs, and cats. Journal Clin Microbiol 43: 22562260 .

\section{Asociación Cooperadora de la Facultad de Ciencias Veterinarias Universidad Nacional del Nordeste}

Personería Jurídica $\mathrm{N}^{\circ}$ 647/92 y 912/00

Sargento Cabral 2139

3400 Corrientes

Argentina

La Asociación Cooperadora de la Facultad de Ciencias Veterinarias de la UNNE fue constituida el 10 de diciembre del año 1991 como entidad de bien público, con el objeto de promover y coadyuvar las actividades científicas, educativas y culturales relacionadas con las Ciencias Veterinarias. En tal sentido, implementa acciones para colaborar con la enseñanza, extensión, actualización y difusión científica que realiza dicha casa de estudios.

\section{Beneficios que brinda a sus asociados:}

- Fotocopias con descuentos especiales en la Fotocopiadora Copias.com que funciona dentro del predio de la Facultad de Ciencias Veterinarias.

- Descuentos para la adquisición de libros de distintas editoriales.

- Descuentos especiales en otros rubros.

Tel. (0379) 4425753 interno 186 • Página Web www.vet.unne.edu.ar • E-mail: cooperadora@vet.unne.edu.ar 